FM1:

Dynamical problems in Extrasolar planets science 


\title{
Dynamical Problems in Extrasolar Planetary Science
}

\author{
Alessandro Morbidelli ${ }^{1}$ and Nader Haghighipour ${ }^{2}$ \\ ${ }^{1}$ Laboratory Lagrange, UCA, CNRS, OCA, Nice, France \\ email: morby@oca.eu; \\ ${ }^{2}$ Institute for Astronomy, University of Hawaii-Manoa, HI \\ email: nader@ifa.hawaii.edu
}

The past few years have witnessed a large increase in the number of extrasolar planets. Thanks to successful surveys from the ground and from space, there are now over 1000 confirmed exoplanets and more then 3000 planetary candidates. More than 130 of these systems host multiple planets. Many of these systems demonstrate physical and orbital characteristics fundamentally different from those of our solar system. The challenges associated with the diversity of planetary systems have raised many interesting questions on planet formation and orbital dynamics.

The list of dynamical problems in extrasolar planetary science is long. Planets in mean motion resonances prompt the investigation of resonant dynamics in the framework of the general (i.e. non-restricted) three-body problem. The general problem is much more complicated than the restricted one, so that we have yet to achieve a global description of resonant dynamics. For instance, previous observations have identified several systems of giant planets in mean-motion resonances, whereas recent observations point to systems of small planets which seem to lie outside the resonance libration domains. While the physics of the processes governing planet-disk interactions suggest a perfect capture in a mean-motion resonance during planetary migration, the reason for these near-resonance configurations is not yet fully understood. Tidal interactions could have played a role in extracting planets from perfect resonance capture, and so might have planetesimal scattering or turbulence in the original proto-planetary disk.

The origin of "hot" exoplanets (planets with orbital periods of a few days) is also another mystery in extrasolar planetary science. It is still a matter of debate whether these planets reached their orbits by migrating through the proto-planetary disk, or they arrived at their current orbital configurations via scattering and tidal damping. Another unresolved problem is the origin of the surprisingly large eccentricities and/or inclinations (relative to the stellar equator) of many extrasolar planets. While planet instabilities, planet-disk interactions, and external perturbations from eccentric or inclined stars have been presented as viable options, the debate is still open on what is the dominant mechanism for the origin of these large eccentricities and inclinations.

In addition to addressing the above-mentioned problems, dynamical models also complement observations by allowing a better characterization of extrasolar planets. Dynamical maps have been very useful to constrain the orbits of multi-planet systems for which the uncertainties in the orbital parameters due to the observational errors are often much larger than the range of orbital configurations ensuring the long-term stability of the system. Another important phenomenon that is a characteristic of planet-planet interactions in multi-planet systems is Transit Time Variation (TTV). TTVs are now routinely detected in these systems and are used to determine mass of planetary bodies and confirm planet candidates. The power and success of this technique can be emphasized by recent discoveries and orbital determination of non-transiting planets through 
the analysis of the TTV signals of their transiting companions. The TTV method brings Celestial Mechanics back to the glorious time when Le Verrier and Adams predicted independently the existence and the position of Neptune from the analysis of the anomalies of the motion of Uranus. Last but not least, dynamical studies are essential to determine long-term orbital stability. Assessing planet stability is necessary to determine whether specific planets can maintain their orbits in the habitable zone for long durations of time, necessary for the development and evolution of life, to understand the long-term properties of their spin etc..

The 2015 IAU General Assembly provided a very timely occasion to organize a meeting on the advancements made in extrasolar planetary science with a focus on the formation, evolution, and characteristics of multiple planetary systems. This focus meeting was timely in the sense that it followed the end of the primary mission of the Kepler space telescope and the discovery of several thousands planets and planetary candidates. The aim of the focus meeting was to have a diverse scientific program, covering all topics related to planetary dynamics in multiple planetary systems and the challenges associated with them. The topic covered by the focus meeting included

- Planet formation;

- Planet migration;

- Secular dynamics and planet instabilities;

- Spin orbit alignment/misalignment;

- Mean-motion resonances and packed planetary systems;

- Transit time variations;

- Tidal evolution, and

- planets in binary systems.

The program consisted of a series of invited talks (each session had at least one invited talk) as well as a rich collection of contributed oral and poster presentations.

The collection of papers presented in this section is a representative of the diversity of the contributions that animated the meeting. The paper by Tan et al. proposes a new scenario of inside-out formation of planets, potentially capable of explaining the abundance of super-Earth planets on short-period orbits. Strugarek et al. and Klahr \& Lobo Gomes present results of their studies of the effects of magnetic torques and convective over-stability on planet migration. Planet migration is also the subject of the contribution by Wang \& Jianghui who investigate the distribution of orbital period ratios in the multi-planet systems observed by the Kepler telescope. The lack of a strong concentration of the orbital period ratios around resonant values is discussed by Chatterjee et al. and explained as a result of planetesimal scattering in the aftermath of gas removal. The paper by Antoniadou \& Voyatzis investigates which configurations allow long-term stability in systems with planets on eccentric orbits, and papers by Jontof-Hutter et al. and Barros et al. describe the most recent results obtained from TTV analyses on $\mathrm{Ke}$ pler multi-planet systems. Tidal evolution of planetary systems are discussed in several papers. Ferraz-Mello et al. discuss a model focused on the tidal evolution of stars with planets and Hirano et al. present a more observationally orientated analysis, reporting on a search for close-in planets around evolved stars which has the potential of testing tidal decay models. The paper by Naoz reviews the dynamics of planets in binary star systems and Campante et al. present a new method to assess the spin-orbit alignment of planets using asteroseismology.

As can be imagined, the organization of such a focused meeting and the preparation of its proceedings chapter is a large task beyond the capabilities of only two people. We had the pleasure of working with a great team of scientists who did not hesitate to help us at any stage of the work. We are indebted to the Scientific Organizing Committee: E. Ford, 
S. Ida, J. Laskar, A. Levavalier des Etains, R. Mardling, T.A. Mictchenko, C. Terquem and J.L. Zhou, for helping us with the organization of this meeting, beginning from the preparation of the original proposal, to the selection of speakers, and to refereeing the papers for these proceedings. We are also thankful to many anonymous referees for their help and willingness to review all submitted articles. Finally, we would like to thank the authors for their participation in this focus meeting, their willingness to contribute to these proceedings, and for their cooperation and responsiveness during the editorial process.

We hope that this chapter, although only a representative of a fraction of what was presented at the meeting, will contribute to the intellectual stimulations and ongoing discussions on the fascinating problem of the origin of planetary systems.

Alessandro Morbidelli (Former President of Commission 7: Celestial Mechanics and Dynamical Astronomy)

Nader Haghighipour (President of IAU Division F: Planetary Systems and Bioastronomy) 